\title{
Mitral Valve Replacement Surgery in Redo Patients with Mix Blood Cardioplegia (MBC) Protection
}

\author{
Saimir Kuci ${ }^{1} \quad$ Ermal Likaj $^{2} \quad$ Alfred Ibrahim $^{1} \quad$ Jonela Burimi $^{1} \quad$ Andi Kacani $^{2} \quad$ Edvin Prifti $^{2}$ \\ 1. Mother Teresa University Hospital Center, Department of Cardiac Anaesthesia \\ 2. Mother Teresa University Hospital Center, Department of Cardiac Surgery \\ Adress: Rr. Dibres 372 Tirana, Albania
}

\begin{abstract}
Aim of Study: Redo patient undergoing valve surgery are always a challenge for both cardiac surgeon and anesthesiologists. Mix blood cardioplegia has had a profound impact on cardiac surgery. but there have been few studies on its use in mitral valve replacement, especially in redo patient. The purpose of this study was to determine whether mix blood cardioplegia offers any advantages in redo patient undergoing mitral valve replacement.Patients and Methods: 20 patient, who have had a previous mitral valve surgery and were scheduled for mitral valve replacement with or without tricuspid repair, were randomized retrospectively to one of two groups of 10 with different technique of myocardial protection: group A (10 patients) had cold crystalloid cardioplegia, and group B(10 patients) had mix blood cardioplegia, a technique modified on our clinic condition (made by mixing 400-500ml oxygenated blood from oxygenator and $10 \mathrm{ml} \mathrm{KCL} \mathrm{7.5 \% ).} \mathrm{Systemic} \mathrm{hypothermia} \mathrm{was} 28^{\circ} \mathrm{C}$ in Group A and between $32^{\circ} \mathrm{C}$ and $33^{\circ} \mathrm{C}$ in Group B. The results were primarily assessed on the basis of clinical outcome, such as hematocrit level intra and post CPB, maximum dose of inotropic support, spontaneous rhythm recovery after aortic cross clamping, length of intensive care unit stay and secondly on postoperative blood loss and blood requirements. Results: There were no preoperative or operative differences between the groups with regard to age, sex, diagnosis, rhythm, New York Heart Association functional class, left ventricular ejection fraction, estimated pulmonary artery systolic pressure, operation, or duration of the operation, CPB, or aortic cross clamping and the time when the patients underwent the first operation. There was one death in group A $(10 \%$ mortality). The changes in hematocrit level differs significantly between the two groups on the post CPB period $(p=0,02)$ There appeared to be a trend towards better spontaneous recovery of sinus rhythm after removal of the aortic cross clamp in group B compared with group A, the difference did reach statistical significance. $(p=0.002)$. Patients on group A required more inotropic support than Group B $(p=0.005)$. There were differences even on blood requirements postoperatively, more dominant these requirement were in group A ( $p=0.02)$.Discussion: Mix Blood Cardioplegia had beneficial effects in clinical outcome in redo patients undergoing mitral valve replacement surgery. This may be due to its better preservation of high-energy phosphates and endogenous amino acids, less anerobic metabolic activity on reperfusion, reduced release of cardiac troponin $\mathrm{T}$, and improved post-ischemic functional recovery.
\end{abstract}

Keywords: Miocardial protection, cardiopulmonary bypass CPB, Mix Blood Cardioplegia (MBC)

DOI: $10.7176 / \mathrm{ALST} / 82-04$

Publication date:October $31^{\text {st }} 2020$

\section{Introduction}

Miocardial protection during cardiac surgery is the key point in this kind of intervention. The different techniques of myocardial protection has been evolved in the course of the years, making cardiac surgery more safe. This study is part of several years follow-up in our clinic of cardiac surgery. From many years we had made efforts to change the cardioplegia solution from crystalloid into blood, based on western clinics guidelines and modified on the conditions of our clinic. From 5 years we use successfully warm blood cardioplegia and the results are very good.

Blood cardioplegia has had a profound impact on cardiac surgery, especially in coronary artery bypass surgery, but there have been few studies on its use in mitral valve replacement, especially in redo patient. Warm blood cardioplegia is used to modify reperfusion injury, resulting in improved postoperative contractile function and decreased mortality. The purpose of this study was to determine whether mix warm blood cardioplegia offers any advantages in redo patients undergoing mitral valve replacement. We designed a study to compare 2 techniques of myocardial protection: intermittent crystalloid cardioplegia, and intermittent mix warm blood cardioplegia. The results were primarily assessed on the basis of clinical outcome, and postoperative blood loss and blood requirements.

\section{Patients and methods}

Between January 2018 and September 2020, in Cardiac Surgery Clinic in "Mother Teresa" Hospital Center, Tirana, Albania, 20 patients, who have had previous mitral valve surgery, were scheduled for MVR with or without tricuspid repair, were randomized retrospectively to one of two groups of 10 with different technique of myocardial protection. In Group A (10 patients) intermittent antegrade cold crystalloid cardioplegia (4 grade C) was used. In 
group B, (10 patients) mix warm blood cardioplegia (MWBC) was used. Anesthesia technique were the same in both groups, general anesthesia.

All operations were performed using cardiopulmonary bypass (CPB) with ascending aortic and bicaval cannulation. Systemic hypothermia was $28^{\circ} \mathrm{C}$ in Group A and between $32^{\circ} \mathrm{C}$ and $33^{\circ} \mathrm{C}$ in Group B.

Antegrade cold crystalloid cardioplegia was injected immediately after aortic cross clamping at $15-20 \mathrm{~mL} \cdot \mathrm{kg}-$ 1. and than at 30minutes interval. In Group B the technique MWBC was realized with our modifications on the condition of our clinic, the first dose was cold crystalloid $(15-120 \mathrm{ml} / \mathrm{kg} /$ weight $)$, after 30 minutes a second dose of cardioplegia was made by mixing $400-500 \mathrm{ml}$ oxygenated blood from oxygenator and $10 \mathrm{ml} \mathrm{KCL} 7.5 \%$, and a third dose of the same solution after 20 minutes. The MWBC temperature was gradually increased from $33^{\circ} \mathrm{C}$ to $36^{\circ} \mathrm{C}$ by the end of the infusion. Electrical defibrillation was applied if ventricular fibrillation persisted beyond 2 min after aortic de clamping, and a temporary pacemaker was used if there was no spontaneous rhythm or if the patient's heart rate was less than 50 beats 'min-1. After the operation, if systolic blood pressure was lower than 90 $\mathrm{mm} \mathrm{Hg}$ and urine output less than $1 \mathrm{~mL} \cdot \mathrm{kg}-1 \cdot \mathrm{h}-1$ with central venous pressure between 10 and $12 \mathrm{~mm} \mathrm{Hg}$, inotropic support was started. Our first choice of inotropic agent was adrenaline.

Table 1. Preoperative and Operative Data in Patients Undergoing Mitral Valve Replacement

\begin{tabular}{|c|c|c|c|}
\hline Variable & Group $A(n=10)$ & Group B $(n=10)$ & P-Value \\
\hline Age (years) & $43+/-10$ & $44+/-10$ & 0.32 \\
\hline Sex (male/female) & $7 / 3$ & $8 / 2$ & \\
\hline \multicolumn{4}{|l|}{ Diagnosis } \\
\hline MS & 5 & 7 & \\
\hline \multicolumn{4}{|l|}{ MR } \\
\hline Mixed MS/MR & 2 & 1 & \\
\hline $\mathrm{MR}+\mathrm{TR}$ & 3 & 2 & \\
\hline \multicolumn{4}{|l|}{ Rhythm } \\
\hline Sinus rhythm & 3 & 5 & \\
\hline Atrial fibrillation & 7 & 5 & \\
\hline \multicolumn{4}{|l|}{ NYHA class } \\
\hline \multicolumn{4}{|c|}{ II } \\
\hline III & 9 & 8 & \\
\hline IV & 1 & 2 & \\
\hline PASP (mm Hg) & $60+/-23$ & $63+/-19$ & 0.62 \\
\hline Ejection fraction $(\%)$ & $50+/-7$ & $52+/-5$ & 0.15 \\
\hline \multicolumn{4}{|l|}{ Operation } \\
\hline MVR & 7 & 8 & \\
\hline MVR + tricuspid repair & 3 & 2 & \\
\hline CPB time (min) & $65+/-5$ & $60+/-5$ & 0.45 \\
\hline Aortic cross clamp time (min) & $50+/-5$ & $55+/-5$ & 0.44 \\
\hline Temperature during CPB (c) & $28^{\circ} \mathrm{C}$ & $32-33^{\circ} \mathrm{C}$ & \\
\hline
\end{tabular}

$C P B=$ cardiopulmonary bypass, NYHA = New York Heart Association, PASP = pulmonary artery systolic pressure, $M R=$ mitral regurgitation, $M S=$ mitral stenosis, $M V R=$ mitral valve replacement,$T R=$ tricuspid regurgitation.

Intraoperative and postoperative variables were used to assess primary clinical outcomes including hematocrit levels pre $\mathrm{CPB}$, intra and post $\mathrm{CPB}$ period, spontaneous rhythm recovery after aortic de clamping (no requirement for electrical defibrillation or temporary pacemaker), maximum doses of inotrope, duration of inotropic support, length of intensive care unit stay, postoperative blood loss and blood requirements.

Commutative data are expressed as the mean \pm standard deviation of the mean. Analysis of the difference in clinical outcome between the two groups was performed using Student's t test.

The significance level for differences in all tests was $p<0.05$. Univariate linear regression analysis was used to perform correlation analysis. 
Table 2. Comparison of Clinical Outcomes

\begin{tabular}{lccc}
\hline Clinical Outcome & GroupA $(\mathrm{n}=10)$ & GrupB $(\mathrm{n}=10)$ & p-Value \\
Htc/Pre CPB (\%) & 37.31 & 37.47 & 0.1 \\
Htc/in CPB/post first dosis (\%) & 23.9 & 24.3 & 0.3 \\
Htc/in CPB/post second dosis (\%) & 21.9 & 24.2 & 0.02 \\
Htc/post CPB (\%) & 26.4 & 31.8 & 0.002 \\
Spontaneous rhythm recovery (n) & 1 & 9 & 0.002 \\
Ventriclar fibrilation after aortic declamp (n) & 9 & 1 & 0.007 \\
Reperfusion time (min) & $25+/-5$ & $15+/-5$ & 0.005 \\
Inotropic suport (adrenaline mcg/kg) & $0.08-0.12$ & $0.04-0.08$ & $10 \%($ mortality) \\
Perioperativ death & 1 & 0 & 0.3 \\
& & & 0.06 \\
Intensive care stay (days) & 2.9 & 2.1 & 0.02 \\
Blood loss (ml) & $600+/-100$ & $400+/-100$ & 1.5 \\
Blood transfusion & 2.5 & 10 & \\
Survival & 9 & &
\end{tabular}

\section{Results}

There were no preoperative or operative differences between the groups with regard to age, sex, diagnosis, rhythm, New York Heart Association functional class, left ventricular ejection fraction, estimated pulmonary artery systolic pressure, operation, or duration of the operation, $\mathrm{CPB}$, or aortic cross clamping the time when the patients underwent the first operation. (Table 1). There was one death in group A (10\% mortality). Clinical outcomes are shown in Table 2.

The changes in hematocrit level differs significantly between the two groups on the post CPB period $(p=0,02)$ There appeared to be a trend towards better spontaneous recovery of sinus rhythm after removal of the aortic cross clamp in group B compared with group A, the difference did reach statistical significance. $(\mathrm{p}=0.002)$. According to our protocol for postoperative care, patients in group A required more inotropic support than Group $B(p=0.005)$. There were differences even on blood requirements postoperatively, more dominant these requirements were in group A ( $\mathrm{p}=0.02)$. Postoperative echocardiography showed good left ventricular ejection fractions in both groups. There were no differences between the 2 groups in duration of ICU stays.

\section{Discussion}

The basic concept of MWBC is reduction of myocardial energy demand by maintaining cardiac arrest with hyperkalemic warm blood perfusate during initial reperfusion, to restore high-energy phosphates and enhance cellular repair after ischemic cardiac arrest.

The beneficial effects of MWBC have been studied both experimentally and clinically. They include better preservation of high-energy phosphates and endogenous amino acids, less anerobic metabolic activity on reperfusion, reduced release of cardiac troponin $\mathrm{T}$, and improved post-ischemic functional recovery $\frac{1.5}{\text {. Those }}$ effects facilitate coronary vasodilatation and accelerate early myocardial tissue oxygen saturation during warm reperfusion ${ }^{2}$.

Tenpaku and colleagues demonstrated complete microtubule repolymerization after 10 min of reperfusion with warm blood. This mechanism may be responsible for the early and improved recovery of cardiac function associated with MWBC.

Most clinical studies of MWBC have been undertaken on coronary artery bypass surgery, with more recent investigations on MWBC in congenital heart surgery.

Most results have showed definite advantages in the use of MWBC, although there have been some that showed no beneficial effect of MWBC.

There is some evidence that a glutamate-aspartate supplement to the MWBC (substrate-enriched

cardioplegia) may reduce reperfusion injury and improve both metabolic and myocardial function recovery. Glutamate and aspartate are not available at our institute, so our MWBC was not a substrate-enriched solution, and it is possible that the MWBC benefits would be more obvious if this technique were employed $\stackrel{3.4}{ }$.

Improved spontaneous rhythm recovery has also been observed with the use of MWBC. There appeared to be a trend towards a better cardiac rhythm recovery in the MWBC

group (less requirement of electrical defibrillation and temporary pacemakers), the difference did reach statistical significance when compared with patients in the non-MWBC group.

\section{References}

1. Cohen G, Borger MA, Weisel RD, Rao V. Intraoperative myocardial protection: current trends and future perspectives. Ann Thorac Surg 1999;68:1995-2001. 
2. Nicolini F, Beghi C, Muscari C, Agostinelli A, Maria Budillon A,Spaggiari I, et al. Myocardial protection in adult cardiac surgery: current options and future challenges. Eur J Cardiothorac Surg 2003;24:986-93.

3. Teoh KH, Christakis GT, Weisel RD, Fremes SE, Mickle DA,Romaschin AD, et al. Accelerated myocardial metabolic recovery with terminal warm blood cardioplegia. J Thorac Cardiovasc Surg 1986;91:888-95.

4. Caputo M, Dihmis WC, Bryan AJ, Suleiman MS, Angelini GD.Warm blood hyperkalaemic reperfusion ('hot shot') prevents myocardial substrate derangement in patients undergoing coronary artery bypass surgery. Eur J Cardiothorac Surg 1998;13:559-64.

5. Follette DM, Fey KH, Steed DL, Foglia RP, Buckberg GD. Reducing reperfusion injury with hypocalcemic, hyperkalemic, alkalotic blood during reoxygenation. Surg Forum 1978;29:284-6. 